\title{
BARRERAS FÍSICAS Y ESTRUCTURALES PARA EL TRATAMIENTO ODONTOLÓGICO DE PERSONAS CON DISCAPACIDAD MOTORA
}

\author{
Henry Francisco Gaitán, Est., Freddy Sánchez-Mendoza, Esp., Gretel González-Colmenares*, Ph.D. .1
}

${ }_{1}$ Facultad de Odontología, Universidad Antonio Nariño, Colombia

Recibido: 16 de mayo del 2013. Aprobado: 24 de junio del 2013.

*Autor de correspondencia: Gretel González-Colmenares. Facultad de Odontología, Universidad Antonio Nariño, Cra. 3 E Nº 47a-15 BI 5, teléfono: 3384960 Ext. 112 , Bogotá, Colombia; correo electrónico: gretgonzalez@uan.edu.co

Cómo citar este artículo: Gaitán HF, Sánchez-Mendoza F, González-Colmenares G. Barreras físicas y estructurales para el tratamiento odontológico de personas con discapacidad motora. Rev Nac Odontol. 2013: 9(17): 41-46.

Resumen. Introducción: esta investigación tiene el fin de identificar barreras físicas y estructurales en el acceso a la consulta odontológica de los pacientes con discapacidad motora. Métodos: se realizó un estudio descriptivo con un grupo de 54 personas en condición de discapacidad física, con edades entre 18 y 80 años, a quienes se les realizó una encuesta sobre las barreras físicas de acceso a los consultorios y las trabas estructurales de la unidad odontológica que impidan una atención en salud oral. Resultados: los motivos de consulta odontológica más frecuentes en este grupo fueron el dolor y la consulta de revisión, con el 36\%, respectivamente. El porcentaje de asistencia a la consulta durante el último año fue de 56,7\%. Se reporta la ausencia de ascensores, de pasamanos en las escaleras y de rampas de acceso en los centros odontológicos. La mayoría reportó problemas con la altura del sillón y con la distancia y altura de la escupidera. Conclusiones: son muchas las barreras de acceso, físicas y estructurales, en los centros odontológicos, relacionadas con el espacio antropométrico y con algunas partes específicas de la unidad odontológica que pueden impedir una atención odontológica apropiada, eficaz y de calidad.

Palabras clave: atención odontológica, barreras de acceso, discapacidad.

Physical and Structural Barriers to Dental Treatment Experienced by People with Motor Disabilities

Abstract. Introduction: Identify physical and structural barriers for access to dental appointments for patients with motor disabilities. Materials and methods: A descriptive study was performed on a group of 54 people with physical or motor disability between the ages of 18 and 80 , who completed a questionnaire on physical barriers for access to dental clinics and structural problems that pose an obstacle to oral health care. Results: The most frequent reasons for their last dental visit over the past year were for pain and their regular checkup, accounting for 36\%. 56.7\% had seen a dentist during the year. Most respondents cited lack of elevators, stair railings and ramps at dental clinics and point to problems with the height of the chair and the distance and height of the spittoon. Conclusions: There are many physical and structural barriers at dental clinics, associated with the anthropometric space and specific parts of the dental unit, which can impede appropriate, efficient and quality dental care.

Keywords: dental care, barriers, disability.
Barreiras físicas e estruturais para o tratamento odontológico de pessoas com necessidades especiais motoras

Resumo. Introdução: identificar barreiras físicas e estruturais no acesso à consulta odontológica dos pacientes com necessidades especiais motoras. Materiais e método: realizou-se um estudo descritivo com um grupo de 54 pessoas com necessidades físicas especiais, com idade entre 18 e 80 anos, aos quais foi realizada uma enquete sobre as barreiras físicas de acesso aos consultórios e as travas estruturais da unidade odontológica que impedem um atendimento em saúde oral. Resultados: os motivos de consulta odontológica mais frequentes neste grupo foram a dor e o retorno da consulta, com $36 \%$, respectivamente. A porcentagem de presença na consulta durante o último ano foi de $56,7 \%$. Constata-se a ausência de elevadores, de passa-mão nas escadas e de rampas de acesso nos centros odontológicos. A maioria relatou problemas com a altura da cadeira e com a distância e a altura do esculpidor. Conclusões: são muitas as barreiras de acesso, físicas e estruturais, nos centros odontológicos, relacionadas com o espaço antropométrico e com algumas partes específicas da unidade odontológica que podem impedir um atendimento odontológico apropriado, eficaz e de qualidade.

Palavras-chave: atendimento odontológico, barreiras de acesso, pessoas com necessidades especiais. 


\section{Introducción}

Una persona con discapacidad presenta restricciones en la clase o en la cantidad de actividades que puede realizar, debido a dificultades corrientes causadas por una condición física o mental permanente $[1,2]$. La discapacidad puede ser cognitiva, física o sensorial [3].

Las estadísticas indican que en países como Gran Bretaña hay alrededor de 8,5 millones de personas con discapacidad, de las cuales alrededor de 6,5 millones están en edad de trabajar [4]. En Colombia, para el 2005 la cifra ascendía a alrededor de 2,6 millones de personas [5].

Se puede decir que la discapacidad motriz comprende todas las alteraciones o deficiencias orgánicas del aparato motor o de su funcionamiento que afectan al sistema óseo, articular, nervioso o muscular. Estas personas presentan una clara desventaja en su aparato motor en relación con el promedio en la población, que se va a manifestar en posturas, desplazamientos, coordinaciones y manipulación [6].

La discapacidad motora muchas veces dificulta acciones rutinarias como el cepillado dental, creando problemas de salud oral, los cuales pueden ser significativamente perjudiciales para la calidad de vida del individuo [7].

No en muchos países se ha discutido la necesidad de implementar un marco general para la conceptualización de los problemas de atención en salud con calidad y seguridad para los pacientes con discapacidad [8].

Son muchas las barreras de acceso que pueden afectar la posibilidad de que una persona con discapacidad reciba atención médica u odontológica apropiada [9]. Dentro de estas se encuentran las barreras geográficas (ubicación de los servicios), las financieras (costos de la atención), las organizacionales (falta de citas, largas esperas, transporte), las culturales [10] y algunas más específicas dentro de la atención en la consulta dental, que pueden ser físicas, estructurales y tecnológicas [11].

En algunos estudios se ha reportado que los equipos normales del consultorio médico, como por ejemplo la camilla, son un obstáculo para la atención de personas con movilidad reducida [12]. Igualmente, en la gran mayoría de las consultas dentales los especialistas no están capacitados para atender este tipo de personas $[13,14]$ y no se cuenta con la tecnología y los equipos (unidad odontológica) adecuados que facili- ten el acceso y la atención de estos pacientes, quizás por costo o rentabilidad [10].

El objetivo de esta investigación fue identificar las barreras físicas y estructurales presentes en el acceso a la consulta odontológica por parte de pacientes con discapacidad motora, con el propósito de determinar cuáles son las falencias existentes.

\section{Métodos}

Se realizó un estudio descriptivo con un grupo de 54 personas en condición de discapacidad física o motora, clase dos moderada, según la Clasificación Internacional del Funcionamiento de la Discapacidad y de la Salud, CIF [15]; estas personas contaban entre 18 y 80 años de edad, 29 mujeres y 25 hombres, que recibían atención en las fundaciones Corpoalegría, Arteboca y Arcángeles, de Bogotá. Fueron excluidos pacientes con discapacidad mental, intelectual o sensorial. Los resultados de una revisión de la literatura, una indagación a odontólogos generales y especialistas y una discusión informal con algunos discapacitados sobre el tema fueron elementos utilizados para desarrollar un instrumento tipo cuestionario que permitiera la recolección de la información.

Se desarrolló una prueba piloto con un grupo de discapacitados, quienes estaban fuera del estudio, para asegurar que las preguntas fueran adecuadas, claras, comprensibles y con validez de contenido. Se contó con el permiso de las fundaciones, el consentimiento informado por parte de los pacientes y la aprobación ética del Comité de Trabajo de Grado de la Facultad de Odontología de la Universidad Antonio Nariño.

Se entregó a cada persona seleccionada el cuestionario acompañado del respectivo consentimiento informado, donde se explicaban los detalles del estudio. Se obtuvieron 54 entrevistas cumplimentadas.

El análisis estadístico se hizo con el programa IBM sPss Statistics 2011. Se utilizó la estadística descriptiva, no la paramétrica; se tomaron como variables principales la causa de la discapacidad: problemas al caminar, amputaciones y deficiencia motora (monoplejia, hemiplejia, paraplejia, tetraplejia), la edad y el sexo; las variables de resultado incluyeron las barreras físicas de acceso a los consultorios y las partes de la unidad que pueden ser una barrera estructural para recibir la atención odontológica. 


\section{Resultados}

Se contó con la participación de 54 personas, de las cuales el $53,7 \%$ pertenecía al sexo femenino y el $42,3 \%$ al masculino; la muestra fue dividida en cinco grupos de edad: de 18 a 24; de 25 a 34; de 35 a 44; de 45 a 54 y $\geq 55$ años. En los rangos de edad, la mayor representación de la muestra la tuvo el grupo de $\geq 55$ años con el $35,2 \%$, seguido por el grupo de 25 a 34 años, con el $25,9 \%$. En cuanto a la causa de la discapacidad, se evidenció que la amputación representa el valor más alto con el $72,7 \%$ en el sexo masculino y la deficiencia motora sobresale con el $62,9 \%$ en el femenino (figura 1). La amputación es la causa más sobresaliente en los grupos de 18 a 24 y de 35 a 44 años con el 18,2\%; el 92,6\% de los pacientes vive en el casco urbano; el $77,8 \%$, en casa propia; la condición civil predominante fue la soltería con el $40,7 \%$; el $48,1 \%$ es bachiller; el $46,3 \%$ trabaja como independiente.

\section{Asistencia a la consulta dental}

Para el total de la muestra, los motivos de consulta odontológica más frecuentes fueron el dolor y la revisión, con el $36 \%$ y el $33 \%$, respectivamente (figura 2). Un número moderado de los encuestados (56,7\%) fue atendido en el último año por algún odontólogo. El $63 \%$ de los discapacitados por amputación declaró no haber acudido durante el último año a la consulta dental. El 56,6\% de los pacientes prefiere ir acompañado a la consulta por motivos de movilidad.

\section{Barreras físicas de las consultas dentales}

Las personas encuestadas facilitaron información sobre los diferentes sitios a los que asisten a consulta odontológica, ya sean consultorios particulares, clínicas dentales e IPS, entre otros. La mayoría, independientemente de la causa de discapacidad, edad y sexo,

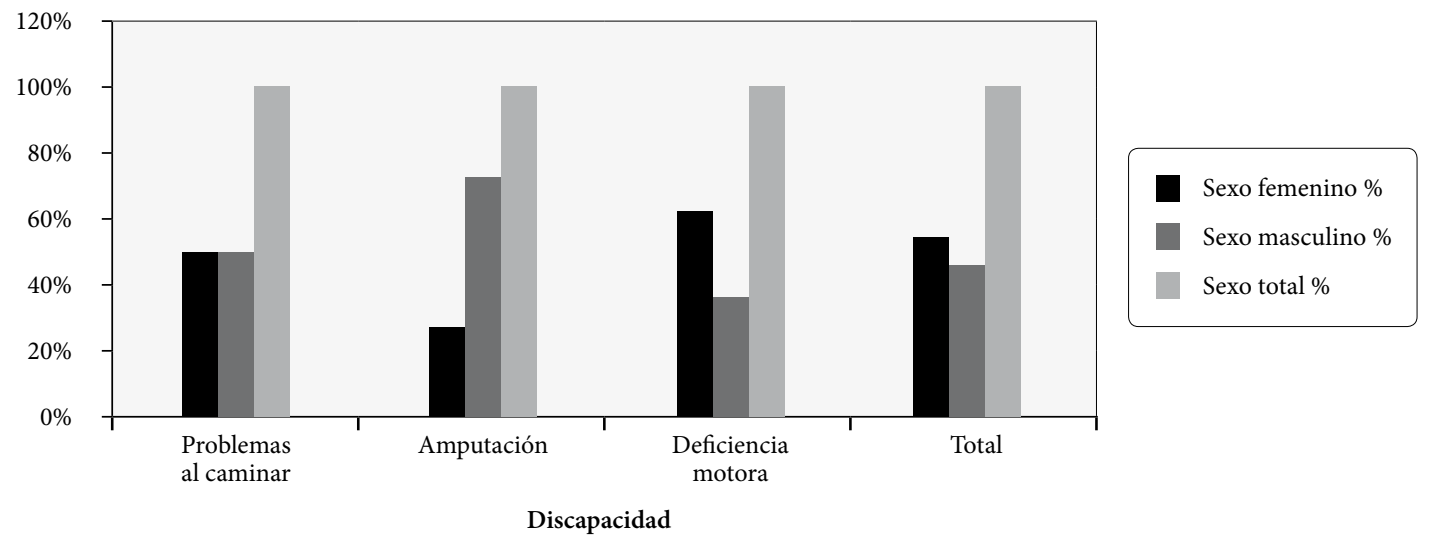

Figura 1. Distribución de la discapacidad por sexo según la muestra

Fuente: elaboración propia. Cálculo y diseño: López, Elizabeth

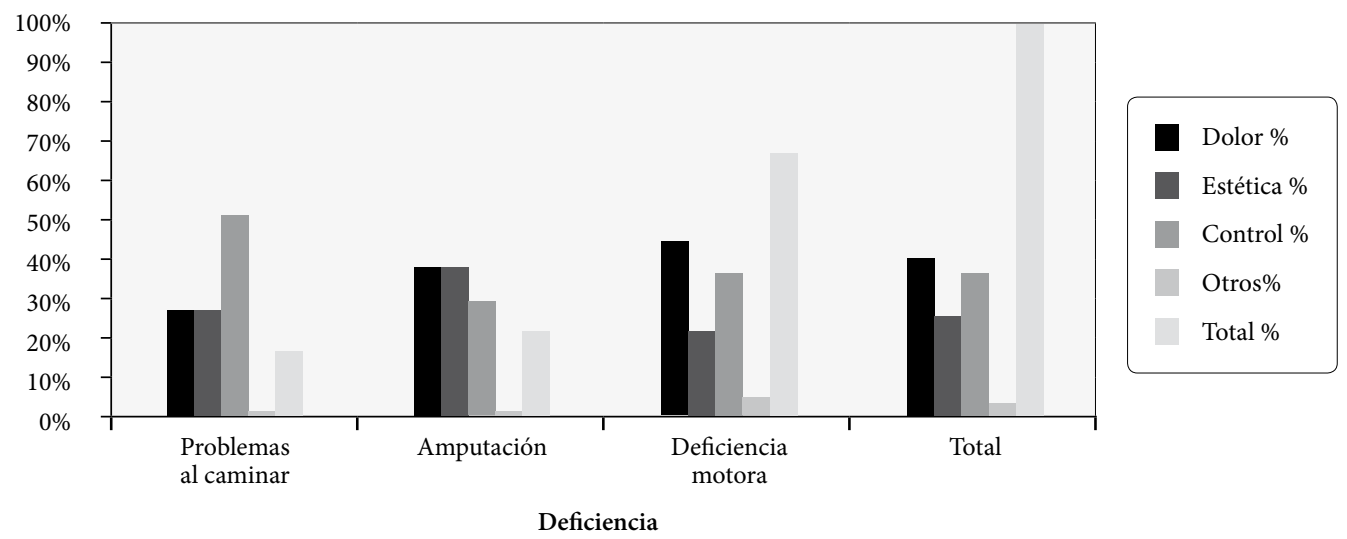

Figura 2. Motivo de la consulta odontológica durante el último año según la causa de la discapacidad Fuente: elaboración propia. Cálculo y diseño: López, Elizabeth 
manifestó dificultades para el ingreso a las instalaciones: el 75,9\% reportó la ausencia de ascensor; el 68,5\%, la ausencia de pasamanos en las escaleras, y el 55,6\% manifestó que no existen rampas de acceso. En cuanto a las plazas de aparcamiento, el 50\% expresó encontrar disponibilidad de aparcamiento para minusválidos. El $70,9 \%$ contestó que no existen baños para discapacitados en los sitios de atención. La mayoría $(85,9 \%)$ reportó que no existen espacios amplios en los centros para el desplazamiento en silla de ruedas (figura 3).

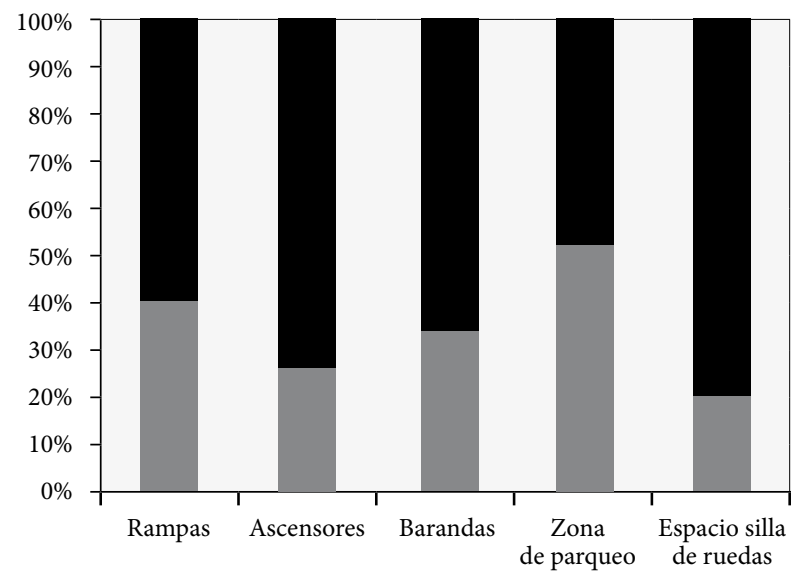

No $\%$ Si $\%$

Figura 3. Barreras físicas de las consultas dentales en el total de la muestra

Fuente: elaboración propia. Cálculo y diseño: López, Elizabeth

\section{Barreras estructurales de la unidad odontológica}

En cuanto a los resultados a los que se refiere la mayoría de las personas con discapacidad motora, en relación con las unidades o sillas odontológicas, se encontró que el 77,8\% de los encuestados manifiesta que el sillón odontológico debería ser más bajo (figura 4); el $83,3 \%$ opina que la distancia y altura de la escupidera no está acorde con sus necesidades; y en cuanto a la ubicación de esta, el 68,5\% consideran que está bien ubicada. El apoyacabeza de la unidad odontológica no le genera confort y no mantiene en posición al $68,5 \%$. La intensidad de la luz de la lámpara no genera molestia para la mayoría $(61,1 \%)$. El 66,7\% refiere que el brazo del sillón se convierte en un obstáculo para el ingreso a la silla odontológica; sólo en el grupo con dificultades para caminar la opinión está dividida (50\%).

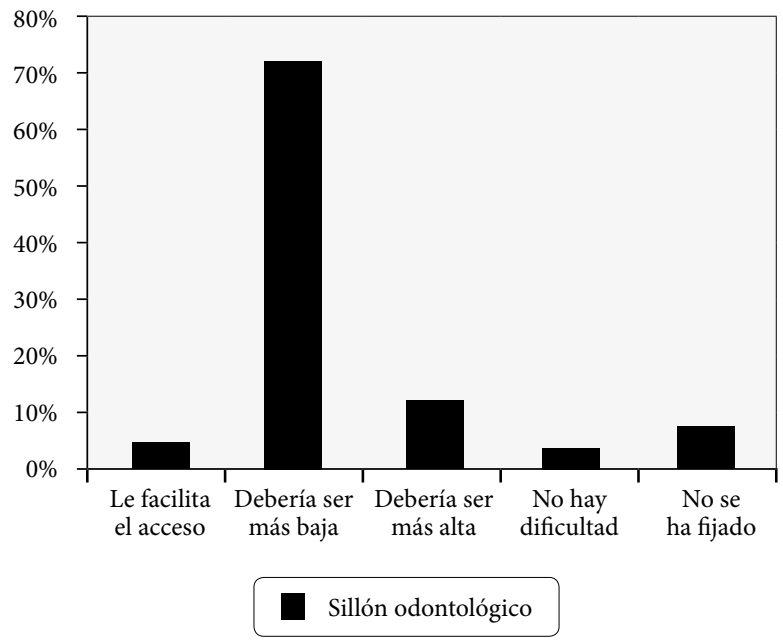

Figura 4. Barreras estructurales de la unidad odontológica. Opinión acerca del sillón

Fuente: elaboración propia. Cálculo y diseño: López, Elizabeth

\section{Discusión}

El objetivo principal de esta investigación era conocer las barreras físicas y estructurales de las personas con discapacidad motora para recibir atención odontológica que esté cercana a ser una atención de calidad. Los sujetos que participaron en este estudio son un pequeño grupo de pacientes con discapacidad motora, pero es indudable que son representativos de las personas que han experimentado bloqueos para recibir una atención odontológica.

En este grupo de discapacitados, la tercera parte son individuos mayores de 55 años, lo que puede estar en relación con lo reportado en Disability Rights Commission Strategic, que identificó que existe un incremento de la incidencia de la discapacidad con el avance de la edad [4].

Para este estudio se agruparon los pacientes de acuerdo con el origen o la causa física de la discapacidad; el primer grupo, con los individuos que aunque tenían presentes sus miembros inferiores y superiores, presentan problemas para realizar una marcha normal; un segundo grupo, en el que se encontraban los pacientes amputados, y un tercer grupo con los pacientes que presentaban deficiencia motora. En la mayoría de los casos los tres grupos coincidieron en sus respuestas; sin embargo, se encontraron algunas diferencias en cuanto a las barreras de infraestructura de la unidad odontológica.

La mitad de los pacientes de la muestra asistió a consulta odontológica en el último año, a pesar de 
sus limitaciones; esto coincide con los resultados de O’Donnell [16] y Rouleau [17].

Se puede visualizar que la salud oral cada vez toma más importancia. Las personas con discapacidad han comenzado a exigir el mismo acceso a la atención odontológica, como la gente sin discapacidad, esperando recibir una atención igual (figura 2).

Casi todos los grupos consideraron que no existía espacio suficiente en el consultorio para acceder con una silla de ruedas, ocasionando que esta se convierta en otra barrera más que impide el acceso al servicio y que se suma a la enfermedad (figura 2).

El Estado colombiano ha buscado garantizar los derechos de la personas con discapacidad. La Ley Estatutaria 1618 de febrero de 2013 pretende mediante "la adopción de medidas de inclusión, acción afirmativa y de ajustes razonables, eliminar toda forma de discriminación por razón de discapacidad"; la normativa incluye la adecuación de instalaciones para adaptar el entorno, con el fin de asegurar el acceso de las personas con discapacidad, en igualdad de condiciones, a los servicios de salud.

Sin embargo, aquí evidenciamos que existen muchas barreras de acceso para los pacientes discapacitados; estas incluyen limitaciones para ingresar a las instalaciones de los consultorios por la ausencia de rampas, ascensores y pasamanos en las escaleras, lo que coincide con las barreras identificadas en los estudios de Bowers y colaboradores [18], y de Grabois [19], quienes las clasifican como barreras físicas y de transporte, de asistencia y de tecnología.

Por lo general, los modelos típicos de atención en salud son inadecuados para las personas discapacitadas [2]. El reto para el sistema de salud es prestar servicios a las personas con discapacidad que sean apropiados, eficientes, eficaces y coordinados, de tal manera que satisfagan sus necesidades, que suelen ser únicas para cada persona de esta población.

La gran mayoría de personas discapacitadas físicamente requiere el cuidado por parte de personal asistente, para su vida diaria y para la atención en otras dependencias, sobre todo si no existe la infraestructura necesaria. Si se brindan servicios de atención personalizada o con herramientas para una atención adecuada, se conseguirá que el individuo discapacitado gane o recupere un poco de independencia [20].

En la mayoría de los casos no basta con eliminar las barreras de tipo arquitectónico, sino que al momento de la atención clínica, la unidad odontológica se convierte en una barrera para el paciente discapacitado.
De por sí, la consulta odontológica, en muchas ocasiones, es una experiencia traumática para las personas, y mucho más para una persona que tiene problemas de movilidad, ya que no sólo tiene que ser trasladada de un lugar a otro, ser sometida al tratamiento, sino que también se tiene que adaptar a una unidad odontológica que no está adecuada a sus necesidades de movilidad.

Las medidas antropométricas son una serie de parámetros preestablecidos que indican unas reglas básicas para tener en cuenta en la construcción o disposición de elementos para el uso humano, como pueden ser sillas, mesas, aparatos sanitarios, etc. Cuando se diseña y construye pensando en las personas con discapacidad, se logran entornos accesibles para todos, con dimensiones adecuadas de los espacios habitables, necesarios para el desplazamiento y maniobra de personas que utilizan sillas de ruedas, muletas, andaderas, bastones y perros guía. Esto, junto con una capacitación universitaria sobre la atención y manejo de las personas con discapacidad, permitirá que los profesionales de la salud identifiquen las barreras de acceso a la consulta odontológica y adecuen su infraestructura.

\section{Conclusiones}

En los consultorios o clínicas odontológicas se evidencian barreras relacionadas con el espacio antropométrico que ocupa el paciente con discapacidad motora; no presentan rampas, ascensores, ni elementos que faciliten su ingreso. Las unidades odontológicas necesitan algunas modificaciones en sus aditamentos, para que el paciente con discapacidad motora pueda acceder al servicio odontológico con plena comodidad y seguridad.

\section{Agradecimientos}

Los autores agradecemos a los pacientes y directivos de las Fundaciones Corpoalegría, Arteboca y Arcángeles, por su participación en este estudio. También al doctor Juan Carlos León, por su colaboración desinteresada.

\section{Referencias}

[1] Organización Mundial de la Salud. Grupo de clasificación, evaluación, encuestas y terminología. Clasificación 
internacional del funcionamiento, de la discapacidad y de la salud (CIDDM-2). Ginebra: oms; 2001. p. 74-81.

[2] Merry AJ, Edwards DM. Disability Part 1: Disability discrimination act (1995)-implications for dentists. Br Dent J. 2002; 193: 199-201.

[3] Penagos LJ. Identificación de barreras físicas de acceso a las clínicas odontológicas para pacientes en condición de discapacidad física en la Universidad Antonio Nariño, sede Bogotá y propuesta de solución. [Trabajo de grado]. Bogotá: Universidad Antonio Nariño, Facultad de Odontología; 2011.

[4] Disability Rights Commission. Change by advice, conciliation and legal enforcement. Strategic Plan 20012004. Londres: Disability Rights Commission; 2004.

[5] Colombia. Departamento Administrativo Nacional de Estadística. Boletín Censo General. Discapacidad. Bogotá: DANE; 2005.

[6] Benítez CR, Hidalgo C. Recomendaciones discapacidad, guía de orientación. Universidad de Las Palmas de Gran Canaria. Cap. 4. Discapacidad motora. [Internet] [consultado el 12 de septiembre de 2012]. Disponible en http://www.ffp.ulpgc.es/desktop/pdfs/vqJgqkTRF0jBJ7fV.pdf

[7] Baird WO, McGrother C, Abrams KR, Dugmore C and Jackson RJ. Factors that influence the dental pattern and maintenance of oral health for people with multiple sclerosis. BR Dent J. 2007; 202: E4.

[8] Blumenthal D. Quality of care-what is it? -part one of six. N Engl J Med. 1996; 335: 891-4.

[9] Hennequin M, Faulks D, Roux D. Accuracy of estimation of dental treatment need in special care patients. J Dent. 2000; 28(2): 131-6.

[10] Lawthers AG, Pransky GS, Peterson LE, Himmelstein $\mathrm{JH}$. Rethinking quality in the context of persons with disability. Int J Qual Health C. 2003; 15(4): 287-99.
[11] Maestre C. The use of general anesthesia for tooth extraction in young handicapped adults in France. Br Dent J. 1996; 180: 297-302.

[12] Welner SL, Foley CC, Nosek MA, Holmes A. Practical considerations in the performance of physical examinations on women with disabilities. Obstet Gynecol Surv. 1999; 54: 457-62.

[13] Cumella S, Rasford N, Lyons J, Burnham H. Needs for oral care among people with intellectual disability not in contact with community dental services. J Intellect Disabil Res. 2000; 44: 45-52.

[14] Pradhan A, Slade G, Spencer A. Access to dental care among adults with physical and intellectual disabilities: residence factors. Aus Dent J. 2009; 54(3): 204-11.

[15] Organización Mundial de la Salud. Organización Panamericana de la Salud. Clasificación internacional del funcionamiento de la discapacidad y de la salud cIF. Madrid: oms; 2001.

[16] O'Donnell D. Barriers to dental treatment experienced by a group of physically handicapped adults in Hertfordshire, England. Quintessence Int. 1985; 16(3): 225-8.

[17] Rouleau T, Harrington A, Bockenek W, Hammond F, Hirsch M, Nussbaum $M$ et al. Receipt of dental care and barriers encountered by persons with disabilities. Spec Care Dent. 2011; 31(2): 63-7.

[18] Bowers B, Joyce M, Esmond S. Quality care: the perspectives of individuals with physical disabilities and their caregivers. Madison, WI: University of Wisconsin-Madison; 1996.

[19] Grabois EW, Nosek MA, Rossi CD. Accessibility of primary care physician's offices for people with disabilities. An analysis of compliance with the Americans Disabilities Act. Arch Fam Med. 1999; 8: 44-51.

[20] Prince JM, Manley MS and Whiteneck GG. Self-managed versus agency-provided personal assistance care for individuals with high level tetraplegia. Arch Phys Med Rehabil. 1995; 76: 919-23. 